\title{
The Mesocosmos: The Success/failure System
}

\author{
Dong-Yih Bau
}

\author{
Department of Information Management, Da-Yeh University, 168 University Rd., Dacun, \\ Changhua 51591, Taiwan, R.O.C.
}

\begin{abstract}
Einstein developed the concepts of principle theory and the cosmos. The principle theory approach is a scientific method that guides scientists to define the structure of the (empirical) universe in logical unity, with the final product called a principle theory. The cosmos is understood as a single logical system that includes three harmonious cosmic views: the microcosmos, macrocosmos, and mesocosmos. These may be respectively considered as the components of the universe defined by quantum mechanics, general relativity, and success/failure system. Herein, we elucidate the concepts of principle theory and the cosmos to explain the mesocosmos, the success/failure system, and the latter's significance for science and humanity. The success/failure system, a principle theory that we recently developed to reflect the mesocosmos, is a twenty-first century scientific discovery. Through the present work, we invite the scientific community to research the mesocosmos and the cosmos on the scale of the universe, in addition to all disciplines of science and final theory currently investigated.
\end{abstract}

Keywords: Einstein, Final theory, Principle theory, Scientific discovery, Structure of the universe, Success/failure system, The cosmos, Totality of existence

\section{Introduction}

The spirit of science is to understand aspects of the universe or the universe (the totality of existence) itself. In the history of science, Einstein was the first to address science on the scale of the universe. His ability to handle this largest scale of things earned praise from Russell, who argued that "In an age when physics has produced a large number of great men and a bewildering variety of new facts and theories, Einstein remains supreme in the breadth and depth and comprehensiveness of his constructions."1:529 Einstein's scientific achievements include general relativity, unified field theory, principle theory, and the cosmos. ${ }^{1,2}$ Whereas the scientific community has received general relativity very well and are familiar with his failure to find a unified field theory, which subsequently pioneered the development of final theory in physics, few have discussed his later achievements in principle theory and the cosmos and his conception of science, which can be found in the literature. ${ }^{1-5}$

Recently, we used Einstein's principle theory to identify the success/failure system principle and develop the success/failure system hypothesis, which reflects the mesoscopic structure of the universe, also called the mesocosmos. ${ }^{3}$ Then, we applied the success/failure system to our understanding of the cosmos, proposing that the theoretical framework of the cosmos or a final theory of the universe may need to incorporate the success/failure system as a third pillar, in addition to quantum mechanics and general relativity. ${ }^{4}$ Then, we conducted a study on the logic of the success/failure system $^{5}$ using Einstein's principle theory ${ }^{1,2}$ and Russell's analytic philosophy. ${ }^{6-9}$ To invite the scientific community to research the mesocosmos and the cosmos, this paper summarizes our theoretical work on the mesocosmos and the success/failure system by elucidating Einstein's concepts of principle theory and the cosmos. Our present work is based on its predecessors, ${ }^{3-5}$ which we suggest examining before studying this paper.

\section{Einstein's Principle Theory and the Cosmos}

The success/failure system, our application of principle theory to the mesocosmos, is a twenty-firstcentury scientific discovery. ${ }^{3-5}$ Since truth stands the test of experience, ${ }^{1: 452}$ we invite the reader to experience building the success/failure system. ${ }^{5}$ This has led us to the decision to clarify Einstein's principle theory and the cosmos. ${ }^{1,2}$ Principle theory is a scientific approach that can be used to solve the problem of the cosmos, in contrast to disciplinary research, which solves problems in a specific field. ${ }^{1,2}$

\subsection{Principle Theory}

Understanding the universe is an incredible task of which disciplinary scientists experience only fragments. Principle theory is a cosmic analysis $\operatorname{method}^{1: 368,369 ; 2: 228}$ that Einstein invented to help render this seemingly incomprehensible universe modestly comprehensible. ${ }^{2: 292}$ In other words, an application of the method must have a direct connection with the empirical world. ${ }^{1 ; 2: 292}$ Thus, a 
scientific discovery employing this method must only be a first-order scientific discovery, rather than second-order or higher-order theoretical constructions, which move farther and farther from the empirical world. This is what Einstein meant by "Experience is the alpha and the omega of all our knowledge of reality."2:271

While the complex universe works how it wants to and will not be dictated to, principle theory guides scientists to build the logical structure of the empirical universe accordingly. ${ }^{1,2}$ Nature or the universe is the sole authority, and academic affiliations, human experts, and journal rankings are irrelevant. The requirement of logical simplicity for cosmic analysis ${ }^{1: 344}$ may cause confusion for many scientists, because only several lines are needed to express the logic, but the scope of study is as wide as the scale of the universe. ${ }^{1-5}$ Furthermore, principle theory requires that "nature is the realization of the simplest conceivable mathematical ideas." 1:385;2:274 The success/failure system uses the discrete mathematical structure, Partial Ordering (PO) conditions for success = Partial Ordering $(\mathrm{PO})$ causes of failure, as in Fig. 1 in our first work, ${ }^{3}$ to create our understanding of the mesocosmos. Further, Einstein said, "In guiding us in the creation of such an order of sense experiences, success alone is the determining factor." $2: 292$ Almost all scientists in disciplinary research may hold that "In God we trust, all others must bring data." 10 In other words, they consider data and reproducibility as the only sources of high research standards. However, humanity is unable to understand the empirical universe by synthesizing data, regardless of their content and size. Furthermore, in any one empirical research, data require definitions and interpretations, which are essentially thoughts. On the other hand, Einstein said that thoughts (logical constructions) are reproducible following the method of principle theory because the method facilitates a highly constrained cosmic analysis, which limits wishful thinking. ${ }^{1-5}$

Despite the above explanation, one may doubt the capability of principle theory and still believe that everyday life poses the hardest question for scientists. Einstein's principle theory is hard to understand, because we need to experience it in order to understand it. If one cannot create the success/failure system, one may not be able to understand it. Thus, we suggest testing it personally. ${ }^{1-5}$ This suggestion would not be naïve since Einstein said, "The most incomprehensible thing about the universe is that it is comprehensible"1:423 and "It is of great importance that the general public [and the scientific community] be given an opportunity to experience - consciously and intelligently - the efforts and results of scientific research."1:400 For this purpose, the success/failure system is an excellent example. One will have an equal chance to 'see' it through the lens of principle theory, since we all live in the universe at the mesocosmic level. Our universe at the mesoscopic level is empirically an erring universe. ${ }^{5}$ When Einstein expressed that "Then I would have had to pity our dear God. The theory [of general relativity] is correct all the same" $1: 368$ immediately after Eddington's validation in 1919 by observing the bending of light through the solar eclipse on the West African island of Príncipe, he simply wanted to say that he had confidence in general relativity framing our understanding of the macrocosmos due to its success as a principle theory one hundred years ago. $^{2: 228}$

To further understand principle theory, we compare it with other scientific methods in the literature. First, analogy is a practical method often applied in science. We know that as pioneers, Descartes and Bacon proposed the methods of deduction and induction, respectively, although we never saw explicit discussion of the appropriateness of applying these methods to the universe. Since mathematics is a means of scientific thought, given increasingly many new mathematical formulations produced in the literature annually, Einstein cautioned that "As far as the laws of mathematics refer to reality, they are not certain; and as far as they are certain, they do not refer to reality." ${ }^{, 371}$ While Euclidean geometry, a famous axiomatic system, has self-evident axioms, principle theory demands more: axioms must be connected to the empirical world, outside of the axiomatic system itself. ${ }^{5}$ Finally, although Polanyi, ${ }^{11,12}$ as with Einstein, ${ }^{1,2}$ considered that a rational idea of the universe can authoritatively speak for itself, he $\mathrm{e}^{11,12}$ proposed a logic of discovery that simply consisted of "intuition followed by creative imagination discerning gestalt," without a guide to discerning the logical structure of the universe. All of these methods and their combinations are currently employed across scientific disciplines. By contrast, to articulate the principle theory approach, Einstein needed to reflect on a successful experience of scientific discovery on the scale of the universe, such as the scientific discovery of general relativity at the macrocosmic level of the universe. ${ }^{1,2}$

\subsection{The Cosmos}

When and where there is no puzzle, there is no problem. The cosmos is viewed through this perspective by many scientists and even our ancestors. However, Einstein believed that there is a pre-established harmony in the universe, and he wanted to experience the universe as a single significant whole. ${ }^{1 ; 2: 38,226} \mathrm{He}$ asserted that "the cosmic religious experience is the strongest and the noblest driving force behind scientific research."1:330 Thus, his conception of science was the cosmos (and principle theory). The concept of the cosmos can be dated back to ancient Greece, where the ancient Greeks considered the seemingly chaotic universe, the cosmos, to be an order in thought. ${ }^{4}$ Einstein took 
a modest scientific view that the cosmos directly refers to the empirical universe, i.e., the fundamental level of the universe. ${ }^{1,2}$ Thus, this concept of the cosmos would not leave all physical (inanimate and living) things in the empirical universe to disorder. Einstein's cosmos included the three harmonious cosmic views or components of the universe defined by quantum mechanics, general relativity, and success/failure system, respectively: the microcosmos, macrocosmos, and mesocosmos. ${ }^{1-5}$

Einstein believed in the profound truth of quantum mechanics, but considered its expression in terms of statistical laws to be a limitation. ${ }^{1: 381 ; 2}$ Nevertheless, he considered a unifying theoretical basis for quantum mechanics (or quantum theory later on) and general relativity by saying "The theory of relativity, as I developed it originally, still does not explain atomism and the quantum phenomena... This demonstrates that the original formulation of the theory of relativity is not definitive... its means of expression are in [the] process of evolution..."1:394,395;2 Indeed, quantum theory and general relativity are the two great revolutions of twentieth-century modern physics. The proposed unification of quantum mechanics and general relativity, which reflect the microcosmos and macrocosmos, respectively, is called a final theory. ${ }^{4}$ Current solution strategies, such as "string theory" and "quantum gravity," have made some progress towards creating a final theory of our universe. ${ }^{4,5}$ For example, string theory has shown the possibility of integrating general relativity and quantum mechanics. ${ }^{4}$ However, a final theory of our universe can only form a foundation of physics, which involves disciplinary physical research. An understanding of the cosmos demands more; it demands a consideration of the universe as a whole, which includes life. ${ }^{1: 56,80,339 ; 2: 224-226}$

Einstein showed interest in two great mysteries of the universe: (1) "to contemplate the mystery of conscious life perpetuating itself through all eternity"1:330 and (2) "to reflect upon the marvelous structure of the universe which we can dimly perceive."1:330 Einstein solved the second mystery of the universe by creating general relativity, which reflects the macrocosmos. ${ }^{3} \mathrm{He}$ was aware of the mesocosmos as he stated, "Our actions should be based on the ever-present awareness that human beings in their thinking, feeling, and acting are not free but are just as causally bound as the stars in their motion."1:332 However, he was also aware of the difficulty of seeking the mesocosmos: "We have penetrated far less deeply into the regularities obtaining within the realm of living things, but deeply enough nevertheless to sense at least the rule of fixed necessity.... What is still lacking here is a grasp of connections of profound generality, but not a knowledge of order in itself."2:47,48 Thus, he abandoned the problem of the mesocosmos before his death in 1955.

\section{ISSN 2455-6378}

We have solved the problem of the mesocosmos by creating the success/failure system, which we developed by applying Einstein's principle theory. ${ }^{3-5}$ We were among the first to approach the problem of the mesocosmos and to ponder the exceedingly delicate threads that connect sense impressions in their totality at the mesoscopic level of the universe. Doing such research requires inexhaustible patience, perseverance, ${ }^{2: 227}$ and certainly a "kind of cosmic religious feeling." $1: 329$ Thus, the current status of scientific advancement is that, to use the ancient Greek's sense of the term "the cosmos," we have all three cosmic components of the universe but have yet to build up the cosmos using these three components. This can be likened to the situation where we have the individual jigsaw pieces in our hands and can begin to explore and fit together the puzzle. Our understanding of the cosmos must provide a single theoretical framework capable of providing insights into the nature of space, time, all forces, all matter, and the part-whole structure. ${ }^{1-5}$ From Einstein's point of view, ${ }^{1,2}$ the problem of the cosmos is the holy grail of science and causes the one theory that forms the foundation of the whole of science.

The success/failure system, our understanding of the mesocosmos, is an important new scientific discovery itself on the scale of the universe and, as the latestdiscovered cosmic component in the twenty-first century, paves the way for scientific advancement to the ultimate scientific theory, our understanding of the cosmos, with the universe as a whole.

Einstein considered our understanding of the cosmos as "God's thoughts," one of the obscurest terms in science, and the rest as details. ${ }^{1: 324}$ Indeed, the details refer to all disciplines of science currently practiced, including cross-disciplines, inter-disciplines, and multi-disciplines. Disciplinary research accumulates knowledge of some reduced aspects of the universe. We may now immediately attend to the hidden fact that ever since the dawn of humanity, scientific progress has focused on parts of science and not the system of science. It was Einstein, a great thinker among others in science, who prepared for us the concept of the cosmos, which answered what the system of science would be like, in probably the only possible way. ${ }^{1,2}$ The cosmos and disciplinary research should shed light on each other. Disciplinary research may provide some clues to but cannot replace our understanding of the cosmos. We believe that the supreme reason or the most beautiful logic is attributed to nature, the cosmos. ${ }^{1,2}$ Whereas the cosmos tends towards a final product, disciplines of science produce more than one and a half million articles annually, which are difficult to assimilate even disciplinarily, and seem an endless human enterprise. The scientific community may need to set up a priority for the mesocosmos and the cosmos in the twenty-first century. 


\section{Significance for Science and Humanity}

The success/failure system is significant for both science and humanity. This scientific discovery may be fraught with further intimations of an indeterminate range. We hope that the scientific significance of the success/failure system will become ever more broadly manifest. We restrict ourselves to the following discussions: articulation of the problem of the cosmos, creation of new scientific concepts, and a new interpretation framework for the mesocosmos. We also briefly describe the significance of this system for humanity.

\subsection{Scientific Significance}

First, a successful application of principle theory to the mesocosmos has led to an articulation of the problem of the cosmos. ${ }^{3-5}$ Principle theory helps us conduct a rational analysis of the logical structures of the inanimate and animate physical realities connected to sense experiences on the scale of the universe. By seeing a moving universe, general relativity revealed the space-time structure of the universe, which reflects the macrocosmos. ${ }^{4.5}$. By perceiving a jiggling universe, quantum mechanics showed the atomic structure of the universe, which reflects the microcosmos. ${ }^{4,5}$ By experiencing an erring universe, the success/failure system exposed the success/failure structure of the universe, which reflects the mesocosmos. ${ }^{4,5}$ Principle theory gives impartial treatment to empiricism and rationalism on the scale of the universe, overcoming the eternal antithesis between them. ${ }^{1,2,5}$ The fundamental level of the empirical universe can provide us with a basic understanding. This gave rise to Einstein's phrasing of "Cosmic religious feeling,"1:329 "God's thoughts,"1:324 and "Nature's rationality"1:323 to express his rapture about the discovery of general relativity and the proposals of principle theory and the cosmos.

With a successful application of principle theory to the mesocosmos, we become aware that Einstein's cosmos includes three harmonious cosmic components. ${ }^{3-5}$ If we now have an understanding of the three cosmic components, can our understanding of the cosmos with the universe as a whole be far behind? We consider the task of the cosmos as the scientist's collective activity. Current disciplinary physical solution strategies for the final theory, such as "string theory" and "quantum gravity," have engendered the multiverse, which may be either a part of reality requiring intensive study or a part of the human mathematical imagination, and the anthropic principle, which may be either a principle of reality or a part of the solution strategies. ${ }^{3-5}$ In any final theory, we anticipate that our understanding of the cosmos will be necessary, clear, and unique.

\section{ISSN 2455-6378}

Second, the success/failure system has created several new scientific concepts. We propose that the concepts and relations of success, failure, part, whole, conditions for success, and causes of failure may be among the justified and necessary scientific concepts, whether logical or empirical, ${ }^{5}$ from which we can understand the part-whole structure. Einstein said that "The supernational character of scientific concepts and scientific language is due to the fact that they have been set up by the best brains of all countries and all times....Their system of concepts has served as a guide in the bewildering chaos of perceptions so that we learned to grasp general truths from particular observations.",:336,337

Principle theory is a rigorous method for creating scientific concepts. ${ }^{1-5}$ To see how difficult the creation of scientific concepts is, we refer to Einstein's logical time-space structure, which was so different from the absolute time and space employed in Newton's theory of gravitation and in our daily lives that principle theory needed to give new scientific meanings to old concepts. ${ }^{1,2}$ Similarly, the success/failure system generates the part-whole structure, ${ }^{3-5}$ which is connected to sense experience on the scale of the universe and is rigorously defined in logical unity. To refer to a comment from Russell, this is quite different to the bewildering and recurrent variety of part, whole, and system concepts created in the sciences of complexity, system, and chaos (which is the so-called new third culture of science other than physics and biology), ${ }^{13}$ in some other areas of intellectual domains and in our daily lives. Thus, we anticipate that there will be a limited number of logically justifiable and necessary scientific concepts in our understanding of the cosmos. All disciplines of science continue to overgenerate, defensibly as science or not, a growing body of scientific concepts and mathematical equations with endless human interests and conflicts. Third, the success/failure system has developed a basic interpretation framework for the mesocosmos. All disciplinary research concerning aspects of the universe at the mesoscopic level must be careful in relation to data interpretation. For example, when biologists discuss whether there are universal laws in biology, we know that we need to search for them at the level of the mesocosmos rather than in the partial world of disciplinary research. ${ }^{3}$ Similarly, it may be true that although there are no new physical, chemical, and biological laws in astrobiology, ${ }^{14}$ there can still be a universal principle theory governing the mesocosmos. Almost all biologists may counter the Gaia hypothesis that Earth and the living things that it supports form a single organism; ${ }^{15}$ with this basic interpretation framework, Earth is a success/failure system. ${ }^{3}$ Finally, it may be now unwise to say that we live in a random evolutionary world governed by Darwin's theory of evolution, without being aware that we basically live in the mesocosmos or at the mesocosmic level of the universe. 


\subsection{Significance for Humanity}

Our understanding of the cosmos, also called a theory of the universe by Einstein, ${ }^{2}$ forms our basic understanding of the material universe. We need a culture of respecting basic universal principles. Science allows us not only to understand but also to respond. Our current scientific culture tends to follow the concept of exploitation, which leads to a deterioration of the homo-ecosystem. ${ }^{3}$ With our understanding that we live in the mesocosmos, where success/failure systems permeate on Earth, which itself is a success/failure system, our scientific culture should obey the concept of conservation. ${ }^{3}$ Russell considered humanity's future to be determined by science. ${ }^{6}$ Thus, the structure of the universe provides a basic but key understanding of the material universe. Humanity, as explored in art, ethics, philosophy, religion and technology, and even by Einstein, demands more than that which our understanding of the material universe may offer. In this regard, our understanding of the universe should shed light on humanity in its widest sense, which constitutes its highest good. For example, the technological innovation principle basically follows the success/failure system principle. ${ }^{3-5}$

\section{Conclusions}

In the history of science, the three main streams of scientific progress have focused on the following three objects of study: matter, the starry heaven, and life. Satisfyingly, these three main streams can now be understood as the following three levels of the empirical universe: the microcosmos, macrocosmos, and mesocosmos. The fact that the universe is comprehensible is a miracle. Now that humanity has an understanding of all three cosmic components, we can build our understanding of the cosmos as a whole. This paper has organized these ideas around our successful application of principle theory to the mesocosmos, the success/failure system.

In this paper, we stake ourselves on declaring in no uncertain terms that the success/failure system is a new experiential scientific discovery or revolution in the twenty-first century, with cosmic-level scope and Nobel-level importance. Our aim is to attract the scientific community to recognize its importance and conduct research on the mesocosmos and the cosmos. As Einstein said, "In striving to do scientific work, the chance-even for very gifted persons-to achieve something of real value is very small.,"1:405 Our series of work has demonstrated that Einstein's principle theory can be a surrogate for gifted persons to achieve such real value. The success/failure system was created to reflect the mesocosmos, and eventually Einstein's cosmos, the new modern science that challenges our best with the anticipation of new scientific discoveries. From this perspective, we will gaze at the universe with transcendental lucidity, perhaps by the end of this century. A theory of the empirical universe will form a general but basic understanding of the totality of existence and can be shared on Earth, or elsewhere.

\section{Acknowledgments}

The author would like to thank Anthony Abram for editing and proofreading this manuscript. I am grateful to Suzanna Rona for advice and feedback on the manuscript. E-mail: bau@mail.dyu.edu.tw

\section{References}

[1] Calaprice, A. The Ultimate Quotable Einstein Princeton Univ. Press: 56, 80, 323, 324, 329, 330, 332, 339, 344, 368, 369, 371, 381, 385, 394, 395, 400, 405, 423, 452, 529 (2010).

[2] Einstein, A. Ideas and Opinions Bonanza Books: $38,47,48,224-228,271,274,292,336,337$ (1954).

[3] Bau, D. Y. The success/failure system hypothesis. IJASRM 3 (3): 30-34 (2018). http://ijasrm.com/wpcontent/uploads/2018/03/IJASRM_V3S3_496_3 0 34.pdf

[4] Bau, D. Y. The cosmos with the success/failure system. IJASRM 3 (12): 94-97 (2018). http://ijasrm.com/wpcontent/uploads/2018/12/IJASRM_V3S12_1044 94_97.pdf http://vixra.org/pdf/1811.0354v1.pdf

[5] Bau, D. Y. The logic of the success/failure system. IJASRM 4 (2): 254-258 (2019). http://ijasrm.com/wpcontent/uploads/2019/02/IJASRM_V4S2_1199 254 258.pdf http://vixra.org/pdf/1901.0207v1.pdf.

[6] Russell, B. The Problems of Philosophy Project Gutenberg EBook \#5827 (2013).

[7] Russell, B. Human Knowledge: Its Scope and Limits Routledge (2009).

[8] Russell, B. My Philosophical Development Spokesman Russell House (2007).

[9] Russell, B. The Philosophy of Logical Atomism Routledge (2010).

[10] Deming, W. E.: https://academic.oup.com/ije/article/41/6/1503/75 $\underline{0028}$

[11] Polanyi, M. Personal Knowledge: Towards a Post-Critical Philosophy The Univ. of Chicago Press: chs. 11 and 13 (1958).

[12] Polanyi, M. The creative imagination. C\&EN Feature: 85-93 (1966).

[13] Pagels, H. R. The Dreams of Reasons Simon \& Schuster (1988). 
[14] Domagal-Goldman S. D. Wright K. E. Adamala $\mathrm{K}$. et al The astrobiology primer v2.0. Astrobiology 16(8) doi:10.1089/ast.2015.1460 (2016).
[15] Margulis L, Sagan D Microcosmos: Four Billion Years of Evolution from Our Microbial Ancestors. University California Press, Berkeley (1997). 